\title{
Tailored degradation of biocompatible poly(3-hydroxybutyrate- co-3-hydroxyvalerate)/calcium silicate/poly(lactide-co-glycolide) ternary composites: An in vitro study
}

\author{
J. Idaszek ${ }^{\mathrm{a}, \mathrm{b}}$, M. Zinn ${ }^{\mathrm{b}, 1}$, M. Obarzanek-Fojt ${ }^{\mathrm{b}}$, V. Zell $^{\mathrm{b}}$, W. Swieszkowski ${ }^{\mathrm{a}}$, A. Bruinink ${ }^{\mathrm{b}, *}$ \\ a Warsaw University of Technology, Faculty of Materials Science and Engineering, Woloska Street 141, 02-507 Warsaw, Poland \\ ${ }^{\mathrm{b}}$ EMPA, Swiss Federal Laboratories for Materials Science and Technology, Lerchenfeldstrasse 5, 9014 St. Gallen, Switzerland
}

\section{A R T I C L E I N F O}

\section{Article history:}

Received 3 April 2013

Received in revised form 23 May 2013

Accepted 19 June 2013

Available online 28 June 2013

\section{Keywords:}

Composite

Biodegradation

Mechanical properties

Biocompatibility

Mesenchymal stem cell

Polyhydroxyalkanoate

\begin{abstract}
A B S T R A C T
Biodegradable materials, which are currently available for bone tissue regeneration, still have limitations regarding their degradation rate, mechanical stability and/or biological response. Thus, a novel generation of materials for bioactive bone scaffolds is needed that triggers hydroxyapatite formation and can be tailored to suit application-specific requirements. In this study we developed ternary bioactive composite materials composed of poly(3-hydroxybutyrate-co-3-hydroxyvalerate), calcium silicate and poly(lactide-co-glycolide) (PHBV/CS/PLGA), which merged the good bioactivity of CS/PHBV composite and the improved degradation velocity of PHBV/PLGA blend. Bioactive character of all composites was proven by formation of hydroxyapatitelike crystals after already one week of incubation in simulated body fluid. Addition of PLGA significantly increased initial ultimate tensile strength $\left(\mathrm{UTS}_{0}\right)$ and Young's modulus of the ternary composites from $14.3 \pm 1.1 \mathrm{MPa}$ (binary composite) to $22.3 \pm 2.6 \mathrm{MPa}$ and $1.23 \pm 0.05 \mathrm{GPa}$ up to $1.64 \pm 0.14 \mathrm{GPa}$, respectively. Furthermore the degradation rate (measured as a decrease of UTS during degradation) could be successfully tailored and was in range of $-0.033 \mathrm{UTS}_{0}$ to $-0.118 \mathrm{UTS}_{0} \mathrm{MPa} /$ week. The bioacceptance of the materials was proven in vitro using 2-D (conventional setup) and 3-D (multicellular spheroids) human bone marrow stromal cell cultures.
\end{abstract}

(c) 2013 Elsevier B.V. All rights reserved.

\section{Introduction}

The number of critical bone defects is increasing due to change of life style and outdoor activities as well as higher life expectancy of the population [1]. Current methods used to treat those defects have several disadvantages. Grafting of autologous bone, the golden standard in bone repair, is limited by the size of autograft and donor site morbidity [2]. Allo- and xenografts bring the risk of autoimmune rejection as well as transmission of pathogens, while the most commonly used alloplasts, e.g. metallic implants and ceramics, do not match the mechanical properties of the bone, may undergo corrosion under physiological conditions or are brittle [2].

Biodegradable implants are considered as an alternative to the above mentioned therapies. The bioresorbable screws, bone plates, stents or scaffolds for tissue engineering support the damaged site temporarily, allowing bone regeneration, and resorb during and after the new tissue is rebuilt $[3,4]$. Moreover, one of the biggest advantages

\footnotetext{
* Corresponding author at: Materials-Biology Interactions Lab, EMPA, Lerchenfeldstrasse 5, CH-9014 St. Gallen, Switzerland. Tel.: + 4158765 7695; fax: +41 587657788 .

E-mail address: arie.bruinink@empa.ch (A. Bruinink).

${ }^{1}$ Current address: HES-SO Valais, University of Applied Sciences Western Switzerland, Route du Rawyl 64, 1950 Sion, Switzerland.
}

for use of the biodegradable implants is the elimination of need for a second surgery.

The biodegradable devices are made predominantly of the FDAapproved polylactides (PLA), polyglycolide (PGA) and their copolymers (poly(lactide-co-glycolide); PLGA). These polymers are composed of $\alpha$-hydroxy acids and undergo relatively fast bulk erosion [5]. The products of the hydrolytic degradation can be accumulated in the core of a device and released in a burst-like fashion into the surrounding environment. Due to the drop of $\mathrm{pH}$, which in vivo can be as low as 2 units [6], a strong inflammatory reaction can be induced resulting in a local resorption of the bone.

Therefore, polymers with different degradation profiles, such as polyhydroxyalkanoates (PHAs), are currently investigated as possible tissue replacement materials. PHAs are aliphatic polyesters that are produced by many bacteria as an intracellular storage compound of carbon and energy [7]. PHAs are composed of $\beta$-hydroxy acids [7], which are less acidic than $\alpha$-hydroxy acids. A typical representative of thermoplastic PHAs is poly(3-hydroxybutyrate-co-3-hydroxyvalerate) (PHBV) which belongs to the subclass of short-chain-length PHAs. Due to its bioacceptance and biodegradability PHBV is currently applied in tissue engineering [8-10]. PHBV exhibits good mechanical properties [9] and, similarly to bone, is piezoelectric [11]. Moreover, no inflammatory reactions have been reported after in vivo implantation of PHBV [12]. 
However, the PHBV exhibits a relatively long degradation rate in comparison to lactic acid-based polymers [13]. Knowles and Hastings observed no changes in dry weight of PHBV samples during almost 600 days and decrease of number average molecular weight by around $40 \%$ after over 2 years of incubation in vitro $(\mathrm{pH}=$ $7.4,37^{\circ} \mathrm{C}$ ) [14]. Moreover, no apatite layer is formed on the surface of PHBV as noted by Li and Chang [15]. Lack of bone-like apatite layer on the surface of an artificial material may lead to fibrous tissue formation and implant encapsulation in vivo [16,17]. Attempts were undertaken to alter the degradation rate of PHBV by either blending it with faster degrading polymers or inorganic fillers. Blends of PHBV with either PLA [18] or PLGA [19] showed improved degradation but not bioactivity, since those polymers are not bioactive (defining bioactivity as the potency of the material to induce calcium phosphate deposition on its surface) $[17,20]$. The bioactivity of the PHBV may be improved by addition to the polymer matrix of inorganic fillers such as bioglass (BG) [21], tricalcium phosphate (TCP), hydroxyapatite (HA) [22], and mineral form of calcium silicate (CS) - wollastonite $[15,23]$. Li and co-workers reported that proliferation and differentiation of human bone marrow stromal cells (HBMCs) were increased on PHBV-CS composites in a concentration dependent manner (relative to neat PHBV), even in the absence of osteogenic factors in the cell culture medium [24]. However, inorganic particles (among them CS) tend to agglomerate at high concentrations, which negatively affects the mechanical properties (i.e. reduction of the tensile strength and elongation at break) and by that strongly limits the applicability of such composites [20]. Additionally, higher concentrations of CS may lead to an increase of $\mathrm{pH}$ of degradation fluid above the physiological value $[15,23]$. Moreover, the presence of alkaline dissolution products results in decelerated decrease of molecular weight compared to neat polymer $[21,23]$. Therefore we decided to use a low concentration of inorganic filler and tune the degradation of composites by addition of third phase, namely PLGA.

The aim of this study was to fabricate a ternary bioactive composite PHBV/CS/PLGA with tailored degradation rate. The PHBV, binary (PHBV and $\mathrm{CS}$ ), and ternary composite films (PHBV, CS and different concentrations of PLGA) were fabricated by means of solvent casting and incubated in simulated body fluid (SBF) up to five weeks. The effect of PLGA on the degradation rate of the ternary composite was investigated by monitoring changes of mass of specimens, molecular weight, $\mathrm{pH}$ of SBF and surface morphology. A ternary composite with superior mechanical properties and optimal degradation rate was selected and subsequently evaluated regarding its biological acceptance using primary human bone marrow mesenchymal stromal cells (HBMCs).

\section{Materials and methods}

\subsection{Materials}

PHAs (PHBV with $10 \mathrm{~mol} \%$ of 3-hydroxyvalerate (HV) and $1 \mathrm{~mol} \%$ of poly(3-hydroxy-10-undecenoate), PHUE) were extracted from lyophilized biomass using methylene chloride [25]. The biomass was subsequently separated from the polymer solution by filtration and concentrated by means of rotary evaporator. The polymer was then precipitated in excess of cold ethanol $\left(\sim 4{ }^{\circ} \mathrm{C}\right)$, collected by vacuum filtration and dried at $40{ }^{\circ} \mathrm{C}$ and 200 mbar for $24 \mathrm{~h}$. The obtained raw polymer was subsequently washed 3 times in three organic solvents, namely ethanol, acetone and heptane. Purified polymer was stored at $-20^{\circ} \mathrm{C}$.

Poly(D,L-lactide-co-glycolide) (PLGA) with the lactide:glycolide molar ratio of 50:50 (Resomer RG $504 \mathrm{H}$; i.v. $0.45 \mathrm{dl} \cdot \mathrm{g}^{-1}$ ) was purchased from Boehringer-Ingelheim (Germany).

CS was synthesized by means of precipitation method as described by Wei et al. [26]. Briefly, $\mathrm{Ca}\left(\mathrm{NO}_{3}\right)_{2} \cdot 4 \mathrm{H}_{2} \mathrm{O}$ (Fluka Analytical, Germany) was dissolved in deionized water at a final concentration of $0.5 \mathrm{M}$ and thereafter 2\% w/v of Pluronic 123 (P123; Aldrich, USA) was added.
Subsequently, $\mathrm{Na}_{2} \mathrm{SiO}_{3} \cdot 9 \mathrm{H}_{2} \mathrm{O}$ solution (Aldrich Chemistry, Japan) at a concentration of $0.5 \mathrm{M}$ in deionized water was added drop-wise while stirring. The obtained CS precipitate was washed 5 times with deionized water and diluted with dimethylformamide (DMF) (Sigma-Aldrich, Germany). The water was removed by distillation at $120^{\circ} \mathrm{C}$. The CS particles were stored as a suspension in DMF until usage (final concentration $1 \% \mathrm{w} / \mathrm{v}$ ).

\subsection{Preparation of composite films and specimens}

The composite films were prepared by means of solvent casting technique. The PHBV and PLGA (composition given in Table 1) were dissolved in methylene chloride at ambient temperature overnight ( $c=3 \% \mathrm{w} / \mathrm{v}$ ) and filtered through PTFE syringe filter with pore diameter of $1 \mu \mathrm{m}$. Subsequently, the CS suspension was added to the polymer solution, resulting in a final CS concentration of 5 wt.\%. The CS-polymer suspensions were cast into glass Petri dishes with lid $(\mathrm{d}=19.5 \mathrm{~cm})$ and dried in air for 3 days and thereafter under vacuum at $60{ }^{\circ} \mathrm{C}$ for $48 \mathrm{~h}$ to remove remaining DMF. The resulting films were folded, thus they were subsequently dissolved in chloroform, cast again on Petri dishes and dried in air for 5 days and next under vacuum at $40{ }^{\circ} \mathrm{C}$ for $24 \mathrm{~h}$. PHBV films were prepared by dissolution of PHBV directly in chloroform. The as prepared composite and PHBV films were used for further studies. For degradation studies and measurements of mechanical properties dumbbell-shaped samples (length $35 \mathrm{~mm}$, width $4 \mathrm{~mm}$, thickness ca. $0.07 \mathrm{~mm}$ ) were prepared. Circular samples were prepared with a thickness of ca. $0.07 \mathrm{~mm}$ and diameter of $15 \mathrm{~mm}$ for cytotoxicity and cell proliferation tests, and diameter of $6 \mathrm{~mm}$ for cell spreading and outgrowth tests. For preparing the various shapes the blanking technique was used. Specimens were disinfected by immersion in antibiotic-antimitotic solution (AA; Gibco, Invitrogen) for $3 \mathrm{~h}$, washed twice with sterile PBS and either washed one time with or incubated for $18 \mathrm{~h}$ in cell culture medium before use. The preincubation step was performed in order to ensure equilibrium in protein adsorption. Cells were cultured on bottom sides of composite films with flatter and less porous characteristics than top sides (Fig. 1).

\subsection{Degradation study}

The degradation study was carried out in SBF, containing per liter: $7.996 \mathrm{~g} \mathrm{NaCl} ; 0.350 \mathrm{~g} \mathrm{NaHCO}_{3} ; 0.224 \mathrm{~g} \mathrm{KCl} ; 0.228 \mathrm{~g} \mathrm{~K}_{2} \mathrm{HPO}_{4} \cdot 3 \mathrm{H}_{2} \mathrm{O}$; $0.305 \mathrm{~g} \mathrm{MgCl}_{2} \cdot 6 \mathrm{H}_{2} \mathrm{O} ; 40 \mathrm{ml} 1 \mathrm{M} \mathrm{HCl} ; 0.278 \mathrm{~g} \mathrm{CaCl}_{2} ; 0.071 \mathrm{~g} \mathrm{Na}_{2} \mathrm{SO}_{4}$; and $6.057 \mathrm{~g}$ TRIS. The solution was prepared as previously published [27] and $\mathrm{pH}$ was adjusted to 7.4. The specimens were sterilized under UV light and subsequently incubated in $15 \mathrm{ml}$ of SBF at $37{ }^{\circ} \mathrm{C}$ under low tangential agitation for a period of 35 days. The SBF was replaced after $7,14,21$, and 28 days. At each time point on average 5 samples were removed from SBF, rinsed with deionized water, and dried in vacuum oven at $40{ }^{\circ} \mathrm{C}$ for $24 \mathrm{~h}$. Every sample was characterized with respect to change of its mass, mechanical properties, molecular weight, and surface morphology. Additionally, the $\mathrm{pH}$ of the degradation fluid was measured.

\subsubsection{Change of mechanical properties}

The tensile properties were measured using Instron Universal Testing Instrument Model 4502 (Instron Corporation, Norwood, MA,

Table 1

Composition of the evaluated samples.

\begin{tabular}{llll}
\hline Composite & PHBV (wt.\%) & PLGA (wt.\%) & CS (wt.\%) \\
\hline PHBV & 100 & 0 & 0 \\
PHBV95 & 95 & 0 & 5 \\
PHBV90 & 90 & 5 & 5 \\
PHBV85 & 85 & 10 & 5 \\
PHBV75 & 75 & 20 & 5 \\
PHBV65 & 65 & 30 & 5 \\
\hline
\end{tabular}


A

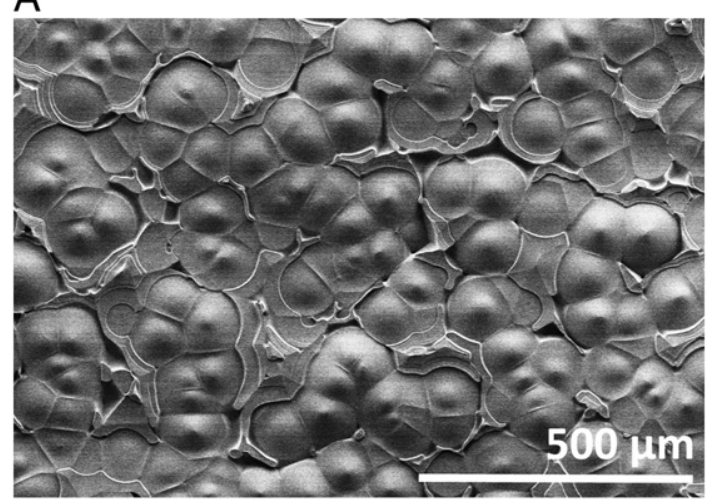

B

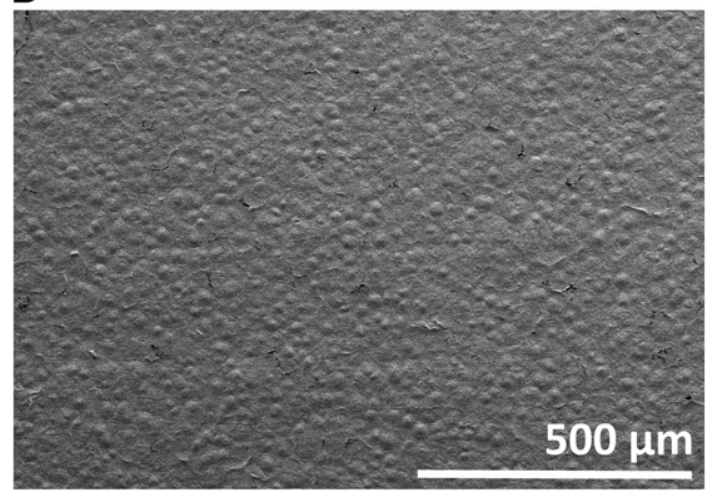

C

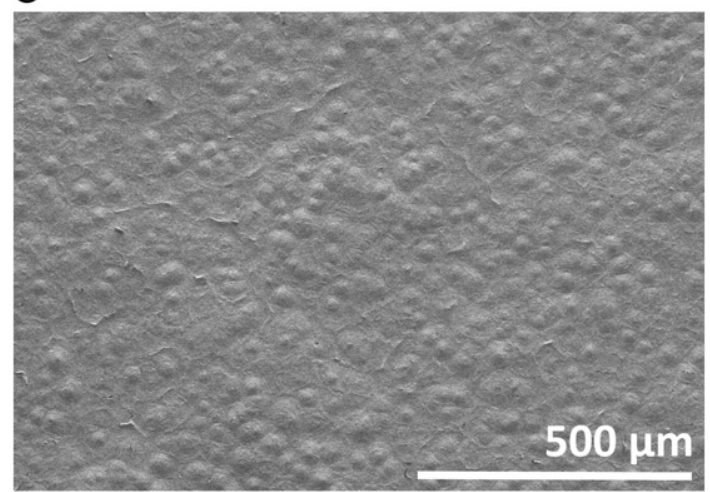

Fig. 1. Scanning electron micrographs of the bottom side of solvent cast films. (A): PHBV, (B): PHBV95, (C): PHBV75. Scale bar $500 \mu \mathrm{m}$.

USA) at $23 \pm 1{ }^{\circ} \mathrm{C}$ and relative humidity $50 \pm 2 \%$. The cross-head speed was $3 \mathrm{~mm} \cdot \mathrm{min}^{-1}$. All samples were preconditioned in the testing atmosphere for at least $24 \mathrm{~h}$ before the test. The ultimate strength $(\sigma)$ was calculated as

$\sigma={ }^{F} / A_{0}$

where: $\mathrm{F}$ - maximal force $(\mathrm{N}) ; \mathrm{A}_{0}$ - initial cross-section area of the sample $\left(\mathrm{mm}^{2}\right)$.The elongation at break $(\varepsilon)$ was calculated as

$\varepsilon=\left({ }^{\mathrm{L}}-\mathrm{L}_{0} / \mathrm{L}_{0}\right) \cdot 100 \%$,

where $\mathrm{L}_{0}$ is initial length of the sample ( $\mathrm{mm}$ ) and $\mathrm{L}$ is length of the sample after elongation ( $\mathrm{mm})$.

The Young's modulus was determined from the initial slope of the linear region of the stress-strain curve (approximately $0.1-0.5 \%$ of strain).

\subsubsection{Change of mass}

Change of the mass was calculated according to formula (3):

$\Delta m=\frac{\left(m_{d}-m_{0}\right)}{m_{0}} \cdot 100 \%$

where: $m_{d}-$ mass of a dry sample after degradation in SBF (after 1, 2, 3,4 and 5 weeks); $m_{0}$ - mass of a dry sample before degradation.

\subsubsection{Change of molecular weight}

Changes of the molecular weight of the specimens were determined by gel permeation chromatography (GPC) using a refractive index detector (GPC apparatus: Agilent, SG). Three specimens of each material were dissolved in HPLC-grade chloroform (POCH, PL) for $36 \mathrm{~h}$ to obtain final concentration of $3 \mathrm{~g} \cdot \mathrm{ml}^{-1}$. Aliquots of $100 \mu \mathrm{l}$ of the polymer solution were injected into chloroform and separated on two linearly coupled SEC columns (PLgel $5 \mu \mathrm{m}$ MIXED-C, UK, $300 \times 7.5 \mathrm{~mm}$ ) at $25{ }^{\circ} \mathrm{C}$ and a flow rate of $0.7 \mathrm{ml} \cdot \mathrm{min}^{-1}$. The system was calibrated using 12 polystyrene standards (PSS, D) with known molecular weights (Mw ranging from $5 \times 10^{2} \mathrm{~g} \cdot \mathrm{mol}^{-1}$ to $2.5 \times 10^{6} \mathrm{~g} \cdot \mathrm{mol}^{-1}$ ).

\subsubsection{Change of surface morphology}

Scanning Electron Microscopy (SEM, Hitachi S-4800 Scanning Electron Microscope, Japan) was employed to study the changes of the surface morphology. Samples were sputtered with gold at $5 \mathrm{~mA}$ and $0.04 \mathrm{mTorr}$ for $5 \mathrm{~min}$, which resulted in an approximately $5 \mathrm{~nm}$ thick coating. The pictures were taken at various magnifications and the acceleration voltage of $2.0-7.0 \mathrm{kV}$.

\subsection{Direct contact in vitro cytotoxicity assay according to ISO 10993-5}

Due to logistic limitations we decided to use only three materials, namely PHBV, PHBV95 and PHBV75, for further biological evaluation. PHBV75 was chosen from the set of the ternary composites due to its superior initial mechanical properties and accelerated, yet providing physical integrity of the samples, degradation rate.

The various materials were evaluated for the release of toxic components according to the ISO10993-5 (2009) contact test. For this 3T3 fibroblast cell line (ECACC No. 85022108, Sir William Dunn School of Pathology, Oxford, UK) cells were seeded at a density of $2.5 \times 10^{5}$ per well of a 6 -well plate in Dulbecco's modified essential medium (DMEM; Sigma) supplemented with $10 \%$ fetal calf serum (FCS; Lonza, BE), 1\% PSN ( $5 \mathrm{mg} \cdot \mathrm{ml}^{-1}$ of penicillin, $5 \mathrm{mg} \cdot \mathrm{ml}^{-1}$ of streptomycin, $10 \mathrm{mg} \cdot \mathrm{ml}^{-1}$ neomycin) and $2 \mathrm{mM}$ L-glutamine (All Gibco, Invitrogen). After $24 \mathrm{~h}$, the specimens were gently placed on top of subconfluent 3T3 cells. The surface of samples covered around $18 \%$ of the culture dish. The glass rings were used to press the polymer samples onto the cell layer in order to prevent floating of films. After $24 \mathrm{~h}$ of incubation cytotoxicity of the materials was assessed both qualitatively, using light microscopic analysis, and quantitatively by means of the MTT assay performed according to Bruinink [28]. Cultures with glass rings were used as controls.

\subsection{In vitro bioacceptance}

Primary human bone marrow mesenchymal stromal stem cells (HBMCs) were obtained from bone marrow of femur head of five patients (42-79 years old) undergoing hip replacement surgery after informed consent. The cells were isolated and expanded as previously described [29]. HBMCs used in experiments were in passage 1 (first passage of cells after isolation of HBMCs from bone marrow) or 2 and were cultured either in expansion medium or osteogenic medium $(\alpha$-MEM supplemented with $10 \%$ FCS, $1 \%$ PSN, $50 \mu \mathrm{M}$ ascorbic acid phosphate (Sigma, Japan), $2 \mathrm{mM} \beta$-glycerophosphate (Sigma, USA) and $10 \mathrm{nM}$ 1,25-dihydroxy-vitamin $\mathrm{D}_{3}$ (Sigma, Israel)). 


\subsection{Cell spreading}

Seven days after cell seeding the HBMC cultures were washed with PBS and fixed with $4 \%$ paraformaldehyde/0.2\% Triton X-100. After blocking in $1 \mathrm{wt}$ \% bovine serum albumin (BSA) in PBS the cells were stained for cell cytoskeletal protein-F-actin (Alexa Fluor 488 Phalloidin Conjugate 1:40, A12379, Molecular Probes, Invitrogen) and focal adhesion protein-vinculin (monoclonal mouse anti-vinculin antibody, 1:300, hVIN-1, V9131, Sigma). After 2 h of incubation with the primary antibody, cells were washed three times with PBS and treated for 30 min with Alexa Fluor 546 goat anti-mouse IgG (1:300, A11030, Molecular Probes, Invitrogen) in 1\% BSA/PBS supplemented with goat serum (1:100). Cell nuclei were stained with Hoechst-33258 $\left(1 \mu \mathrm{g} \cdot \mathrm{ml}^{-1}, 861405\right.$, Sigma-Aldrich) for $10 \mathrm{~min}$ and cells were washed twice with PBS. The samples were kept in PBS and analyzed using a fluorescence microscope (Axio Imager M1, Zeiss, DE).

\subsection{Proliferation of HBMCS}

HBMCs were seeded at a density of $1 \times 10^{4}$ per sample $(d=15 \mathrm{~mm})$ and cultured in the expansion medium at $37{ }^{\circ} \mathrm{C}$ in humidified atmosphere. HBMCs seeded on the cell culture dish were used as control. After $6 \mathrm{~h}$ and 1, 4, and 7 days of incubation the specimens with cells were gently washed twice with warm PBS and placed in new wells containing $500 \mu \mathrm{l}$ of bidest water and frozen at $-20^{\circ} \mathrm{C}$. At the end of the experiment DNA content of all cultures was assessed as described previously [30].

\subsection{Outgrowth of HBMCs from cell spheroids}

Before preparing cell spheroids HBMCs were vitally labeled with DiD dye (D307, Molecular Probes, Invitrogen) as previously described [31]. In order to obtain defined cell spheroids, 15,000 HBMCs suspended in $1 \mathrm{ml}$ of medium were centrifuged in $15 \mathrm{ml}$ falcon tubes at $200 \times \mathrm{g}$ at room temperature for $15 \mathrm{~min}$ and incubated in humidified atmosphere at $37{ }^{\circ} \mathrm{C}$ overnight. Each sample was placed in a well of a 24-well plate. A medium droplet containing one spheroid was gently placed on top of each sample and incubated for $2 \mathrm{~h}$ before adding $1 \mathrm{ml}$ osteogenic medium. Pictures of the cultures were taken at day 7 of incubation using a fluorescence microscope Axio Imager M1 (Zeiss, DE) with 625/655 and 655/750 filters for excitation and emission, respectively. The total surface area covered by cells was determined by connecting the locations of outmost cells of the spreading cell population and measuring the resulting polygon surface area by means of AxioVision rel. 4.8.2 software (Carl Zeiss, Germany). Additionally, the surface of the spheroid core, measured as the surface area covered by cells with the highest cell densities, was assessed by the area with the highest fluorescence.

\subsection{Statistical analysis}

Data are expressed either as a mean \pm standard deviation (SD; material characteristics data) or as a mean \pm standard error of mean (SEM; biological data). The results were evaluated statistically by means of post hoc one way ANOVA with a Tukey-Kramer pair-wise comparison test (KyPlot 2.0 beta 15 software).

\section{Results}

\subsection{Mechanical properties of the prepared composites}

Composites with different contents of PHBV, CS, and PLGA (Table 1) were assessed for their mechanical performance (Table 2). Addition of CS had an adverse effect on the mechanical properties of PHBV and decreased the ultimate strength by $31 \%$, Young's modulus by $10 \%$ and elongation at break by $67 \%$, in comparison to neat PHBV. Addition of PLGA, however, improved strength and stiffness of the composites in general. Maximal values were obtained with 20 wt.\% of PLGA. The strength of PHBV75 was improved by $56 \%$ and the modulus increased by $33 \%$ compared to PHBV95. All composites exhibited lower values for elongation at break in comparison to neat PHBV.

\subsection{Degradation and its effect on mechanical properties}

\subsubsection{Change of mechanical properties}

The ultimate tensile strength (UTS) of neat PHBV remained relatively constant over the degradation period, whereas it was found to continuously decrease for all composites (Fig. 2A-C). Fig. 2A depicts changes of the UTS from week 0 until week 5 . The fastest reduction of ultimate strength was observed for composite PHBV65 followed by PHBV75. After 5 weeks of incubation the strength of the latter composites decreased by $55 \%$ and $39 \%$, respectively. The strength of composite PHBV90 diminished the least (20\% of the initial value). The degradation rate, understood as a decrease of UTS of all composites normalized to the value of ultimate strength at week 0 , is shown in Fig. 3. The rate was calculated as a slope of a linear trend line of values of the UTS at each week of degradation for a given composite using the least squares method. A nearly linear correlation between the concentration of the PLGA and the degradation rate was observed for the composites $\left(R^{2}=\right.$ 0.9418).

In line with the ultimate tensile strength the most pronounced decrease of Young's modulus was observed for PHBV65 and PHBV75 and after 5 weeks of incubation in SBF reached $63 \%$ and $71 \%$ of the initial value (Fig. 2B). The change of the tensile modulus of the rest of materials did not reveal any clear trend.

Elongation at break (Fig. 2C) remained relatively constant over the degradation period for binary composite PHBV95 and ternary composites containing low concentration of PLGA (PHBV90 and PHBV85). The elongation started to increase after 3 weeks for composites PHBV75 and PHBV65. After 5 weeks of incubation it reached values slightly lower than those of the neat PHBV.

\subsubsection{The change of mass}

The change of mass of all tested materials is shown in Fig. 4A. All composites, except PHBV65, gained mass over the time of degradation. At the end of the experiment the mass of composites PHBV95 and 90 increased by around $10 \%$ and PHBV85 by $8.4 \%$. PHBV75 reached maximal mass ( $6.9 \%$ of the initial mass) after 4 weeks and PHBV65 already after 2 weeks (gain of $1.5 \%$ ) of incubation. Thereafter the latter two composites started losing mass, which reached 5.9\% (PHBV75) and $-3.8 \%$ (PHBV65) of their initial mass after 5 weeks.

\subsubsection{Change of $\mathrm{pH}$}

The $\mathrm{pH}$ of SBF oscillated in a range of 7.30-7.48 over the degradation period (Fig. 4B) with an elevated peak observed for PHBV95 after the first week of incubation. The pH of degradation fluid of PHBV75 reached 7.33 after 5 weeks of degradation. The highest decrease in $\mathrm{pH}$ value was observed for PHBV65 ( $\mathrm{pH}=7.30$ ).

Table 2

Initial mechanical properties of the fabricated materials determined in a static tensile test. The data are presented as a mean \pm SD.

\begin{tabular}{llll}
\hline Composite & $\begin{array}{l}\text { Ultimate strength } \\
(\mathrm{MPa})\end{array}$ & $\begin{array}{l}\text { Young's Modulus } \\
(\mathrm{GPa})\end{array}$ & $\begin{array}{l}\text { Elongation at break } \\
(\%)\end{array}$ \\
\hline PHBV & $20.6 \pm 0.8^{*}$ & $1.38 \pm 0.10$ & $8.0 \pm 1.1^{*}$ \\
PHBV95 & $14.3 \pm 1.1^{\#}$ & $1.23 \pm 0.05$ & $2.6 \pm 0.5^{\#}$ \\
PHBV90 & $16.9 \pm 0.2^{\#}$ & $1.28 \pm 0.03$ & $6.7 \pm 1.9^{*}$ \\
PHBV85 & $17.7 \pm 1.7$ & $1.41 \pm 0.04$ & $2.8 \pm 0.4^{\#}$ \\
PHBV75 & $22.3 \pm 2.6^{*}$ & $1.64 \pm 0.14^{*}$, & $3.1 \pm 0.4^{\#}$ \\
PHBV65 & $17.9 \pm 2.9^{*}$ & $1.51 \pm 0.22^{*}$ & $2.4 \pm 0.5^{\#}$ \\
\hline
\end{tabular}

* $\mathrm{p}<0.05$ compared with PHBV95.

\# $\mathrm{p}<0.05$ compared with PHBV. 


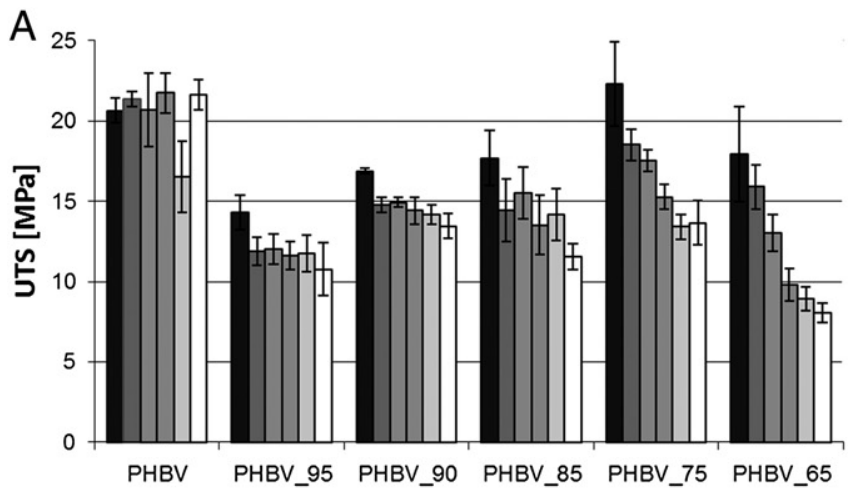

घWeek 0 aWeek 1 aWeek 2 aWeek 3 aWeek 4 aWeek 5

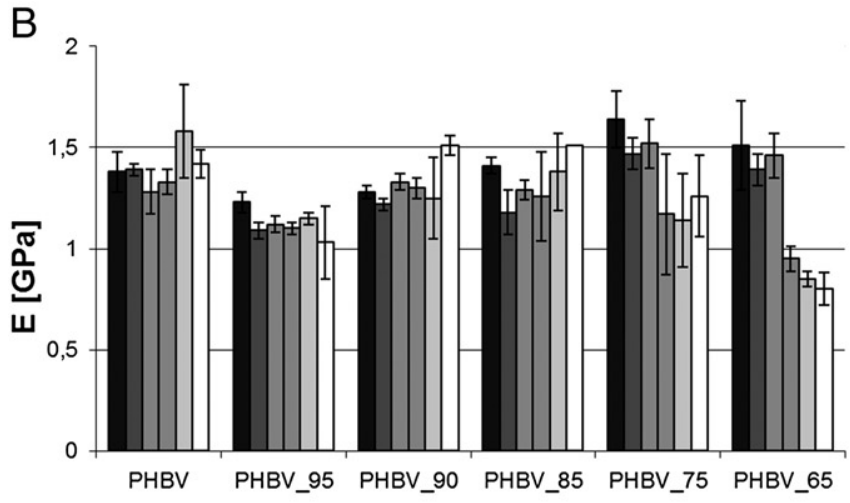

घWeek 0 aWeek 1 aWeek 2 aWeek 3 aWeek 4 aWeek 5

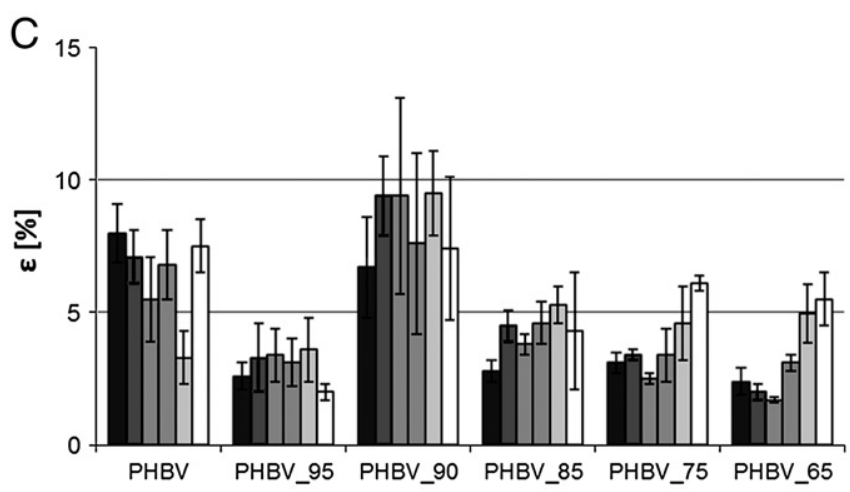

aWeek 0 aWeek 1 aWeek 2 aWeek 3 aWeek 4 aWeek 5

Fig. 2. Change of the ultimate tensile strength (A), Young's modulus (B) and elongation at break $(\mathrm{C})$ of the materials during degradation in SBF as measured up to 5 weeks. The mechanical properties were determined in a static tensile test. Bars represent mean \pm SD.

\subsubsection{Change of molecular weight}

Change of the number average molecular weight $\left(M_{n}\right.$, Fig. $\left.5 A\right)$ of PHBV and PHBV95 exhibited a similar pattern and the value of $\mathrm{M}_{\mathrm{n}}$ oscillated between $212 \pm 27$ and $245 \pm 13 \mathrm{kDa}$. However, $\mathrm{M}_{\mathrm{n}}$ of PHBV75 was significantly lower than $\mathrm{M}_{\mathrm{n}}$ of aforementioned PHBV and PHBV95 due to presence of low-molecular weight fraction of PLGA $\left(M_{n}\right.$ of $\left.\sim 20 \mathrm{kDa}\right)$. The $M_{n}$ of PHBV75 decreased by $25 \%$ during the first two weeks of incubation. From the second week on the $\mathrm{M}_{\mathrm{n}}$ of PHBV75 started to increase, which was accompanied by almost complete disappearance of the PLGA peak in GPC elugram at week no. 3. In case of weight average molecular weight $\left(\mathrm{M}_{\mathrm{w}}\right.$, Fig. $\left.5 \mathrm{~B}\right)$ the highest decrease was observed for PHBV75 (by $48 \%$ of initial value), followed by PHBV95 (by $24 \%$ of initial value) and PHBV (decrease of $M_{w}$ by $15 \%$ of initial value).

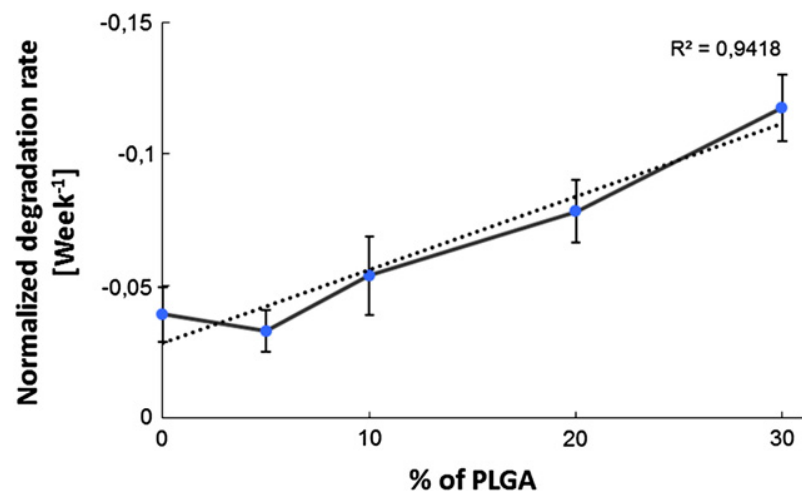

Fig. 3. Correlation between PLGA content and normalized degradation rate of PHBV95PHBV65. Each point represents a slope of a linear trend line of values of the ultimate tensile strength (normalized to strength at week 0 ) at each week of degradation for a given composite calculated using the least squares method.

\subsubsection{Change of the surface morphology}

Cauliflower-like crystals (typical morphology of hydroxyapatite) were observed on the surface of all five composites already at first week of degradation (Fig. 6E and F; PHBV75 is a representative of the set of ternary composites). In line with this observation EDS analysis revealed that these crystals contained Ca (30\%), P (15\%) and O (50\%) (wt.\%) (Fig. S3). No apatite-like crystals were present on the surface of neat PHBV even after five weeks of incubation in SBF (Fig. 6D). The inclusions of PLGA visible on SEM images as hemispheres diminished after first week of degradation. Additionally, formation of submicron voids was observed on PHBV65 composite already after the first week and on PHBV75 composite after 4 weeks. The size of the void kept increasing during subsequent weeks of degradation.

A

A

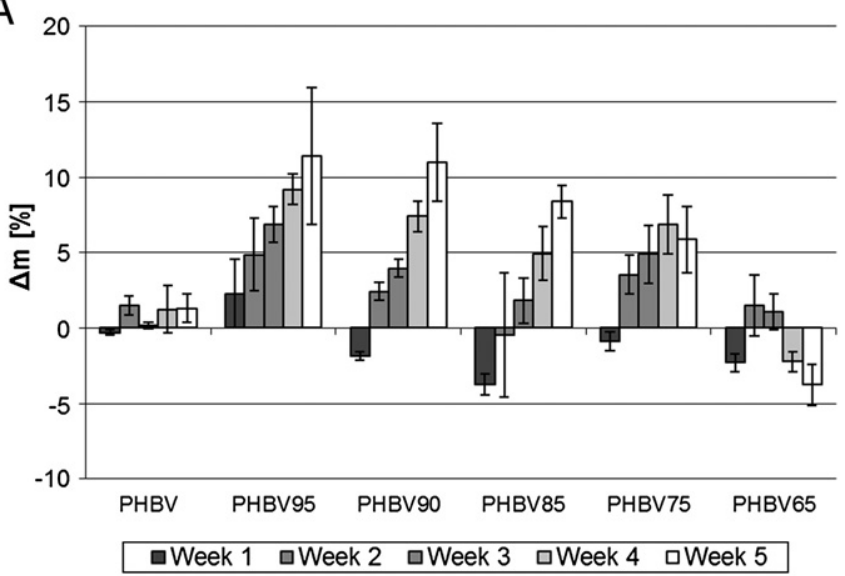

B

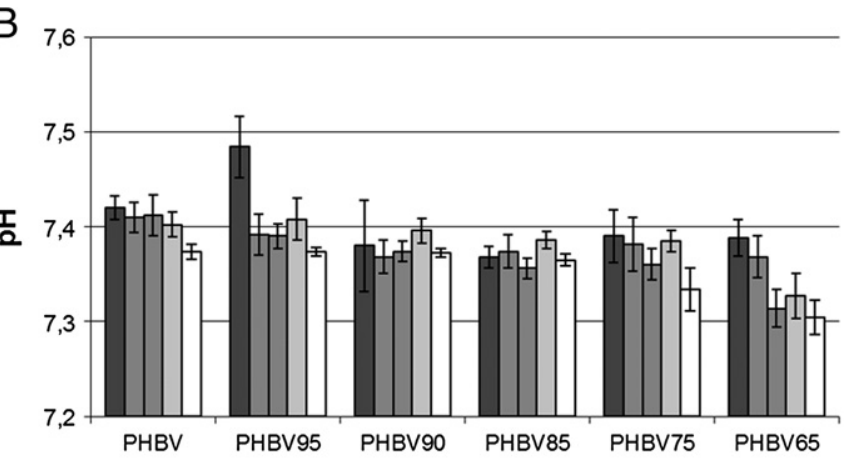

aWeek 1 aWeek 2 aWeek 3 aWeek 4 aWeek 5

Fig. 4. Change of mass of the samples (A) and pH of the SBF (B) during degradation. The $\mathrm{pH}$ was measured at the end of each degradation time point after removing the sample. 

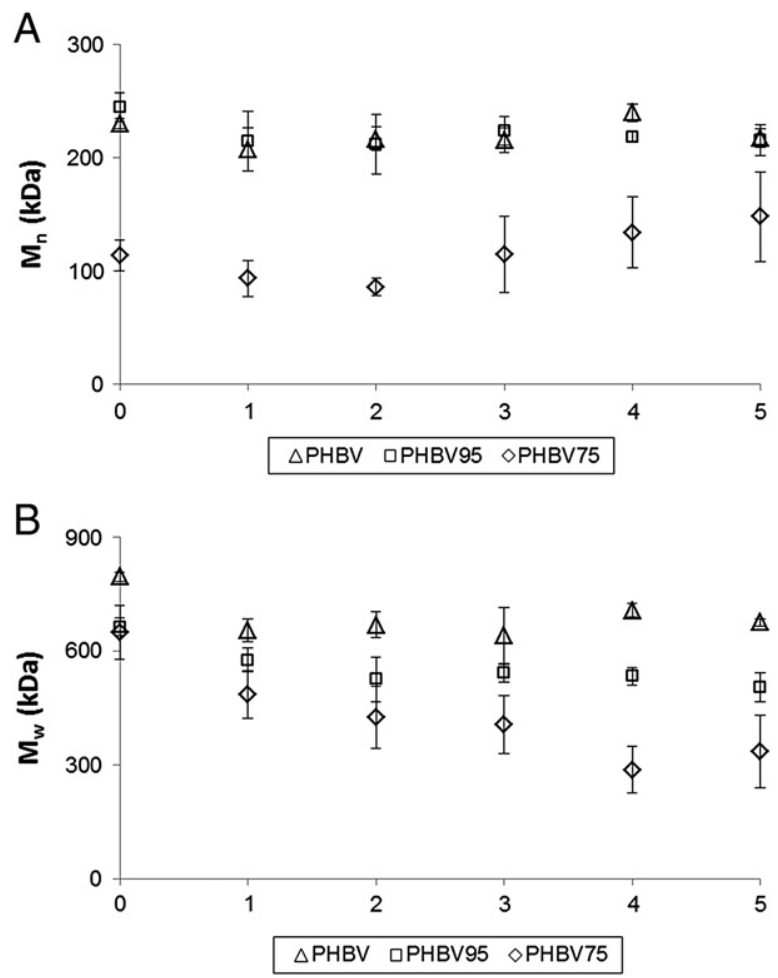

Fig. 5. Change of molecular weight of 3 tested materials during the degradation period of 5 weeks. (A) Number average molecular weight. (B) Weight average molecular weight. Each point represents an average value of the molecular weight of three different specimen \pm SD.

\subsection{ISO 10993-5 direct contact test}

Light microscope images revealed the presence of 3T3 cells in the area surrounding or below the samples. The cells incubated with test materials exhibited a similar morphology to the control cultures (Fig. S1 - Supplementary data). Moreover, no effect of the three tested materials on total cell culture energy state was found (MTT assay, Table 3).

\subsection{Cell spreading}

All tested materials supported HBMCs spreading. As illustrated in Fig. S4, the HBMCs exhibited a well-organized actin cytoskeleton with clear focal adhesion points. The actin filaments were mostly arranged in parallel to the cell orientation. However, the cells cultured on PHBV75 had a more irregular morphology than those cultured on PHBV and PHBV95.

\subsection{Cell attachment and proliferation}

Based on studies of Wachem and co-workers [32] it may be assumed that cell adhesion of seeded cells is completed after $6 \mathrm{~h}$. After this time the number of adherent cells on all materials was not significantly different as measured by total culture DNA (Fig. 7). After $24 \mathrm{~h}$ of cell incubation, the HBMC number was comparable between PHBV and PHBV75, but significantly lower on PHBV95. At day 4 the amount of DNA was approximately 1.5 times higher on all three tested materials when compared to PHBV control measured $6 \mathrm{~h}$ after cell plating. At day 7 the cell number observed on PHBV was 1.4 and 1.3 times higher than on PHBV95 and PHBV75, respectively.

\subsection{Cell outgrowth from spheroids}

The best cell outgrowth from HBMC spheroids after 7 days in culture was observed on PHBV films and reached $14.3 \pm 0.8 \mathrm{~mm}^{2}$ (mean \pm SEM). The outgrowth was lower on both PHBV95 and PHBV75 and reached $11.2 \pm 1.6 \mathrm{~mm}^{2}$ and $10.7 \pm 1.3 \mathrm{~mm}^{2}$, respectively. Additionally, the area with the highest cell density was measured to estimate the flattening of the spheroids. As illustrated in Fig. 8, the core area was the smallest on PHBV $\left(0.21 \pm 0.04 \mathrm{~mm}^{2}\right)$, followed by PHBV75 $\left(0.26 \pm 0.05 \mathrm{~mm}^{2}\right)$ and PHBV95 $\left(0.37 \pm 0.10 \mathrm{~mm}^{2}\right)$.

\section{Discussion}

In the present study a PHBV-CS-PLGA biomaterial was fabricated with special emphasis on improving the degradation behavior without loss of initial mechanical properties and biological acceptance in comparison to pure PHBV material. As a first premise of developing biomaterials it is important to evaluate their biocompatibility (non-toxicity and correct tissue/cell reaction) in vitro. We found that none of the produced and evaluated materials released toxic constituents as measured according to the direct contact test guideline ISO10993-5.

A property of a material "that elicits a specific biological response at the interface of the material, which results in the formation of a bond between the tissue and that material" [33] represents a key parameter for biocompatibility of a material. A non-osteoconductive material (bioinert) can be encapsulated by fibrous tissue, which for biodegradable polymers leads to development of a scar tissue. In case of bioactive bone implants (defined by the potency to induce an apatite layer on its surface), the bonding occurs through a layer of hydroxyl carbonate apatite. Its deposition on surfaces of implants is therefore a highly desired property, especially for biomaterials designed for regeneration of bone tissue. According to Hench a layer of apatite deposited on a surface of a bioactive glass leads to biochemical adsorption of growth factors, followed by attachment, proliferation and differentiation of stem cells, and thus, ongrowth of a new bone tissue [34].

Most of the polymers do not show direct bone apposition and can be enclosed by a fibrous capsule [12,17]. In order to increase bone bonding ability of such materials the concept of bioactive composites has been introduced. It is well known that the addition of certain inorganic fillers leads to precipitation of bone like hydroxyapatite on the surface of the composite [22,15,35]. Formation of a hydroxyapatite layer occurs inter alia in the presence of simulated body fluid (SBF). Therefore, Kokubo et al. proposed SBF as a test solution to predict bioactivity of a material in vitro [16]. As illustrated in Fig. 6, no crystals were present on the surface of PHBV even after 5 weeks of incubation in SBF. However, a dense layer of nanoscale sized calcium phosphate crystals was observed on the surface of all composites already 1 week after incubation in SBF, proving their bioactivity (Fig. S3).

Although the bioactivity of PHBV was positively affected by addition of CS (Fig. 6), the mechanical properties of PHBV95 diminished dramatically, resulting in a composite with the poorest initial UTS and Young's modulus among all studied materials (Table 2). The negative effect of inorganic filler on Young's modulus was already reported by Misra and co-workers in case of solvent cast poly(3-hydroxybutyrate)-based composite films $[35,36]$. The reduction of the mechanical properties of the composite can be explained by agglomeration of the CS particles within the polymer solution and poor adhesion between filler particles and polymer matrix. Agglomerates can act as stress concentrators, which effectively decreases the mechanical properties of the composite $[20,35]$. Weak interactions between the particles and polymer matrix could give rise to a dewetting effect, which additionally decreased the value of the elastic modulus $[35,37]$. Decrease in the elongation at break is a common feature of rigid filler based composites as elongation experienced by the polymer matrix is much greater than the measured elongation of the specimen [37]. 
A

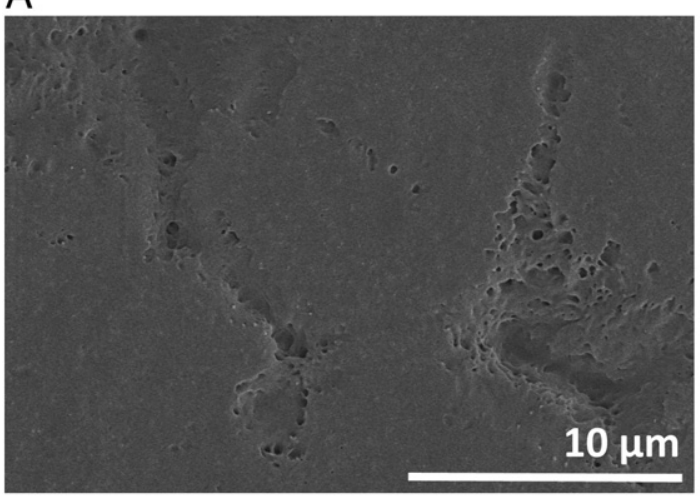

C

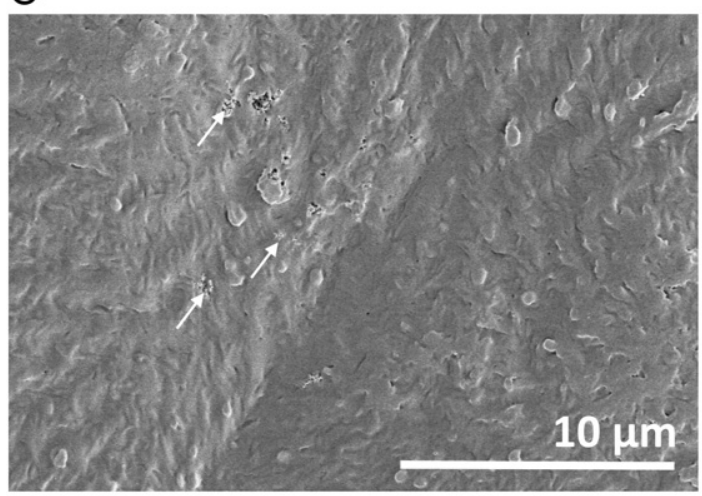

E

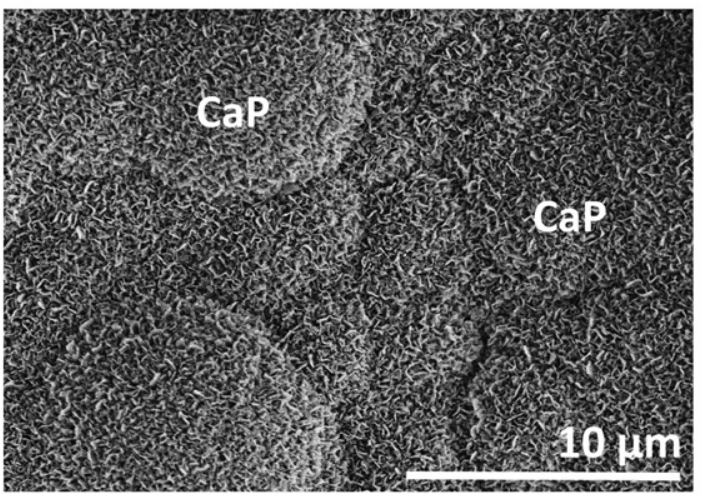

B

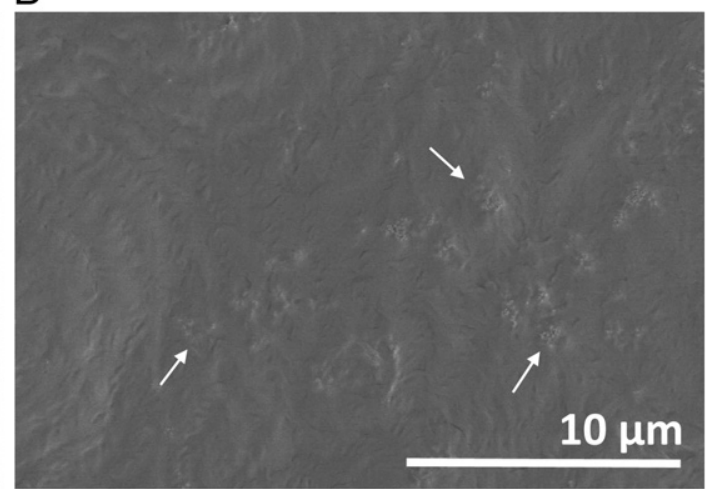

$\mathrm{D}$

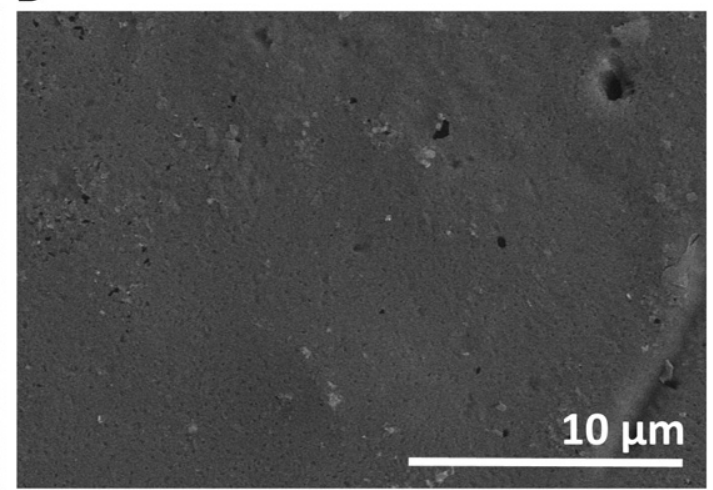

$\mathrm{F}$

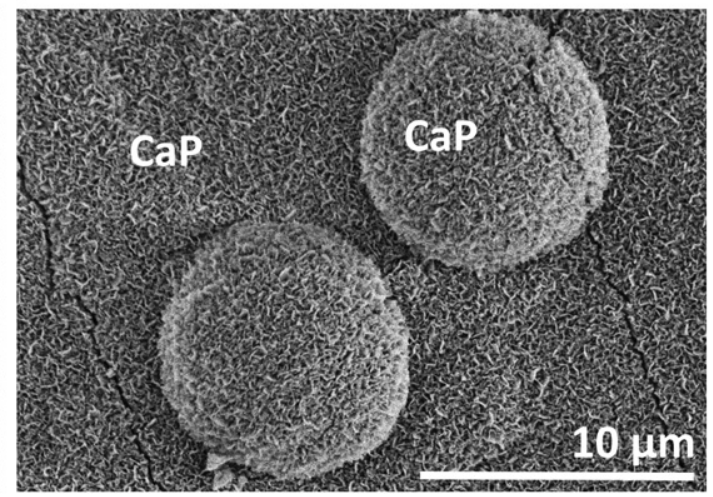

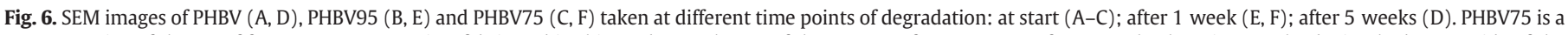

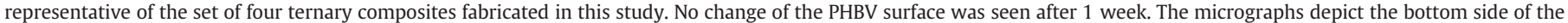
three materials. CaP - calcium phosphate (as determined by EDS). Arrows in parts (B) and (C) indicate some of the CS particles. Scale bar of $10 \mu$ m.

The primary goal, i.e. to increase degradation rate, was poorly accomplished by the addition of CS. As it can be seen in Fig. 2A-C the mechanical properties of PHBV95 remained relatively constant during the degradation period. The drop in ultimate tensile strength after first week of incubation was probably caused by dissolution of CS [26]. Continuous mass gain (Fig. 4A) and relatively constant pH

Table 3

Effects of three selected materials on cell function (MTT conversion activity). MTT-test was performed according to ISO10993-5 cell contact test. Cell cultures were evaluated $24 \mathrm{~h}$ after placing the sample on top of a subconfluent 3T3 cell layer covering around $18 \%$ of the culture. The MTT conversion to formazan relative to control cultures treated with glass rings is presented (mean \pm SD of 3 independent experiments).

\begin{tabular}{llll}
\hline Material & PHBV & PHBV95 & PHBV75 \\
\hline Conversion activity (\% of control) & $99 \pm 6$ & $96 \pm 4$ & $104 \pm 5$ \\
\hline
\end{tabular}

values (Fig. 4B), similar to those of neat PHBV (except week 1), and moderate decrease of $\mathrm{M}_{\mathrm{w}}$ (Fig. 5B) further confirmed the little influence of 5 wt.\% of CS on degradation behavior of PHBV.

In order to accelerate the degradation velocity of PHBV-CS composite, a third phase, i.e. PLGA, was added. The PLGA not only increased degradation rate of the ternary composites but also additionally improved their initial mechanical properties. It may be hypothesized that this reinforcement of PHBV-CS-PLGA composite was due to presence of high modulus inclusions of PLGA ( $E$ in range of $1 \div 4$ GPa [38]). Furthermore, the organic PLGA phase should adhere better to the organic PHBV matrix than the inorganic CS particles. Better adhesion at the interface leads to an effective transfer of the load through the whole composite [37]. Similar effects were observed by Gassner et al. in PCL/PHBV [39] and by Iannace et al. [40] in PHBV/PLA blends.

The influence of PLGA on the degradation properties of the composites is depicted in Fig. 2A-C. In general, the presence of PLGA resulted 


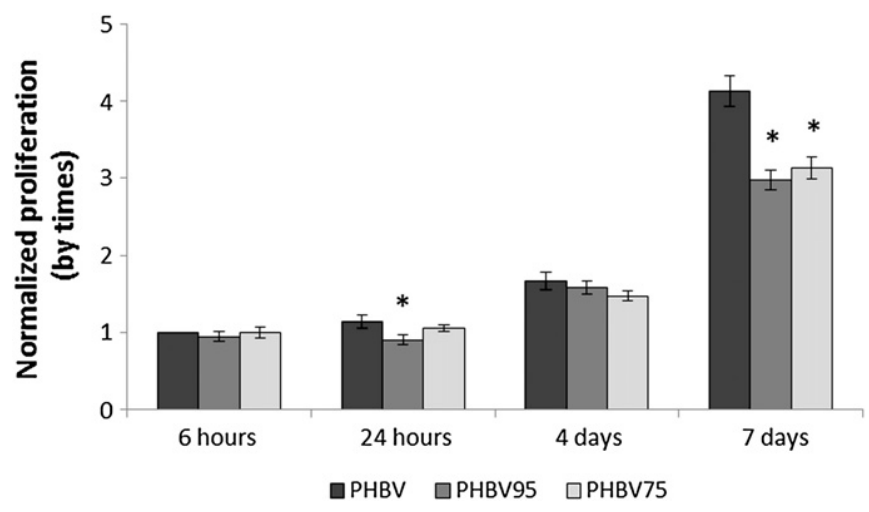

Fig. 7. Time evolution of cell number after seeding of HBMC on PHBV, PHBV95 and PHBV75 samples. The concentration of DNA at different time points was normalized to concentration of DNA on PHBV samples after $6 \mathrm{~h}$ of incubation. The data are presented as a mean \pm SEM over 3 independent experiments. *: $\mathrm{p}<0.05$ compared with PHBV.

in a continuous decrease of ultimate tensile strength of all ternary composite films during five weeks of degradation in SBF (Fig. 2A). However, after an initial deterioration during the first week, the decrease was less pronounced in case of composite PHBV90, which contained the lowest concentration of PLGA. By increasing the PLGA concentration the strength decrease became more prominent. A linear correlation between the concentration of the PLGA and the decrease of ultimate tensile strength was found for all ternary composites (Fig. 3). This finding suggests that the main factor affecting change of the mechanical properties of the composites during the first weeks of incubation in SBF was degradation of PLGA accompanied by a decrease of molecular weight of PHBV (Fig. 5B).

Time evolution of the Young's modulus differed between the composites PHBV95-PHBV85 and PHBV75-PHBV65. After an initial drop, the moduli of the former composites were found to slightly increase while those of the latter kept decreasing. The stiffening could be explained by an increase of crystallinity of PHBV caused by the incubation in SBF at physiological temperature [41]. Similar phenomenon was found for PHBV/PCL blends by Yasin et al. [41]. The authors observed an increase in crystallinity during the first weeks of incubation and concluded that it was caused by chain cleavage in the amorphous phase and its further crystallization. The abovementioned change in mechanical properties was not detected in case of the composites PHBV75 and PHBV65, probably due to an enhanced degradation of PLGA and PHBV (Fig. $5 B$ ). The GPC analysis revealed that $\mathrm{M}_{\mathrm{w}}$ of the ternary composite PHBV75 decreased by nearly $50 \%$ during the time of incubation.

Based on the initial mechanical properties and the degradation profile, we decided to use PHBV75 for further biological evaluation. This ternary composite not only exhibited the best initial strength and stiffness but after five weeks of degradation its mechanical properties were still comparable to those of PHBV95 prior degradation. Additionally, the decrease of molecular weight was more advanced in PHBV75 than in the binary composite, confirming the positive effect of PLGA on degradation of the polymer matrix. Furthermore, the degradation profile of PHBV75 makes it a suitable material to be used in regeneration of bone tissue according to strategy II proposed by Hutmacher [13], in which the scaffold sustains cell proliferation and differentiation up to a moment when the engineered tissue is able to support itself.

The bioacceptance of the materials for bone tissue regeneration was investigated in vitro using HBMCs. As parameters for bioacceptance of the material cell attachment, spreading, and surface colonization during the first days of in vitro culture were evaluated. In this study we found that addition of CS to PHBV did not affect adhesion of HBMCs after $6 \mathrm{~h}$ of incubation, compared to neat PHBV. This is not in line with published data showing that cell attachment to the composite surface may be significantly improved by addition of CS into polyester films [26,42,43]. However, in the present study a much lower filler concentration was
A

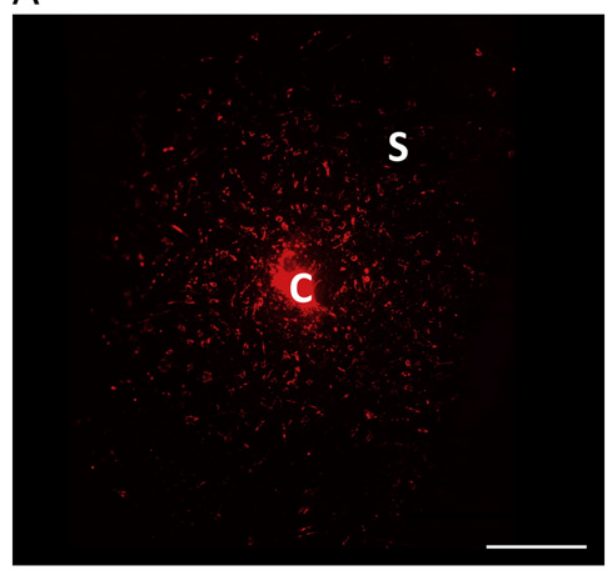

B

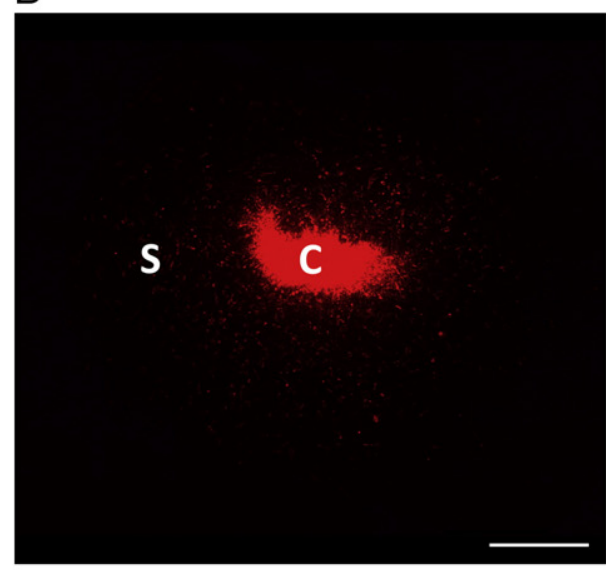

C

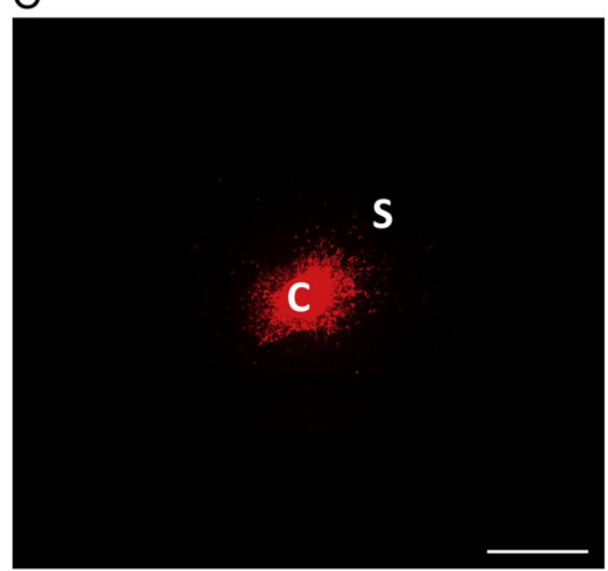

Fig. 8. HBMC outgrowth out of cell spheroids on plane (A) PHBV, (B) PHBV95 and (C) PHBV75 after 7 days in culture. HBMCs were vitally labeled with DiD dye. C: core of the spheroid; S: surface area covered by cells. Scale bar of $1000 \mu \mathrm{m}$.

used in comparison to the latter investigations (5\% vs. 20-40 wt.\% as used by Li et al. [42] and Wei et al. [26]). Next, PHBV95 is slightly more hydrophilic than PHBV (see Supplementary data) and increase in hydrophilicity generally positively affects cell adhesion [44]. Moreover, the cell density of HBMCs was significantly lower on PHBV95 than PHBV after $24 \mathrm{~h}$ and 7 days of culture, respectively. Similar results were reported by Misra and co-workers showing reduced number of MG63 cells on Poly(3-hydroxybutyrate), PHB-BG films with higher filler content [36]. It has been suggested that the reduction may be based on the release of alkaline dissolution products of BG reaching cytotoxic values. However, we could demonstrate that no toxic constituents were released during 
incubation of materials with 3T3 cells (see Fig. S1 and Table 3). Additionally, the HBMCs were well spread on all three materials and exhibited visible actin filaments and focal adhesion points (compare Fig. S4). Furthermore, we did not observe any critical increase of the $\mathrm{pH}$ during first week of incubation in SBF. Therefore our findings suggest that the reported positive effects of CS on cell performance were probably counteracted by factor(s) different from surface chemistry and release of toxic constituents. We hypothesize that one factor is the difference in surface topography (Fig. 1.). The 2D films used by Li [42] were fabricated by compression molding technique and there were no distinctive differences in surface topography between neat polymer and the composites. In the present study the films were produced by solvent casting and exhibited different surface structures and roughness (PHBV: $R_{a}=$ $2.453 \mu \mathrm{m}$; PHBV95: $R_{a}=0.214 \mu \mathrm{m}$; PHBV75: $R_{a}=0.374 \mu \mathrm{m}$; see Table S1, Supplementary data). Addition of PLGA had no significant effect on the attachment and proliferation of HBMCs, when compared to the PHBV95 composite (Fig. 7).

The outgrowth of HBMCs from cell spheroids was analyzed in order to predict the possible host response to the materials. Due to its 3-dimensional (3D) nature spheroids resemble real tissues more appropriately than conventional monolayer cultures [45]. Therefore, Moczulska and coworkers assumed that surface colonization by cells growing out of cell spheroids mimics better the in vivo colonization than by cells seeded as single cells onto the samples (monolayer culture) [46]. In the present study the cell outgrowth out of 3D spheroids composed of HBMCs was evaluated on 2D films. We showed an improved cell outgrowth on neat PHBV relative to the PHBV95 and PHBV75 composites. In line with this finding the highest increase in cell number on neat PHBV was seen after seeding single cells on these materials. However, the appearance of spheroids cultured on neat PHBV differed from those on the composite films (Fig. 8). The latter had a higher core area and less single cells surrounding the core. These differences may be based on the higher surface roughness of PHBV.

\section{Conclusions}

In conclusion, incorporation of CS and PLGA to PHBV matrix allowed us to fabricate bioactive composites with good in vitro bioacceptance, tailored degradation rates and improved mechanical properties. The tailored degradation was proven both at the molecular (decrease of molecular weight) and macroscopic levels (decrease of mechanical properties and weight of the specimens). Addition of PLGA to PHBVbased composites is a suitable method to adjust the degradation rate for an envisioned regeneration strategy without deterioration of the initial mechanical properties of the material. Thus the ternary composite PHBV75 is a promising material for bone regeneration.

\section{Acknowledgments}

This work has been supported by the International PhD school EMPA-Poland project DeSTine and partially by Bioimplant project (GPC, DSC and surface wettability measurements); Grant No. POIG.01.01.02-00-022/09. The stay of Joanna Idaszek at Empa has been supported by the European Union in the framework of European Social Fund through the Warsaw University of Technology Development Programme, realized by Center for Advanced Studies.

\section{Appendix A. Supplementary data}

Supplementary data to this article can be found online at http:// dx.doi.org/10.1016/j.msec.2013.06.025.

\section{References}

[1] C.T. Laurencin, A.M. Ambrosio, M.D. Borden, J.A.J. Cooper, Annu. Rev. Biomed. Eng. 1 (1999) 19-46.

[2] A.J. Salgado, O.P. Coutinho, R.L. Reis, Macromol. Biosci. 4 (2004) 743-765.

[3] J.C. Middleton, A.J. Tipton, Biomaterials 21 (2000) 2335-2346.

[4] L.S. Nair, C.T. Laurencin, Prog. Polym. Sci. 32 (2007) 762-798.

[5] F. von Burkersroda, L. Schedl, A. Göpferich, Biomaterials 23 (2002) 4221-4231.

[6] K. Mader, B. Gallez, K.J. Liu, H.M. Swartz, Biomaterials 17 (1996) 457-461.

[7] M. Zinn, B. Witholt, T. Egli, Adv. Drug Deliv. Rev. 53 (2001) 5-21.

[8] G.A. van der Walle, G.J. de Koning, R.A. Weusthuis, G. Eggink, Adv. Biochem. Eng. Biotechnol. 71 (2001) 263-291.

[9] T. Freier, Adv. Polym. Sci. 203 (2006) 1-61.

[10] S.K. Misra, S.P. Valappil, I. Roy, A.R. Boccaccini, Biomacromolecules 7 (2006) 2249-2258.

[11] Y. Ando, E. Fukada, Int. J. Biol. Macromol. 8 (1986) 361-366.

[12] Z.B. Luklinska, H. Schluckwerder, J. Microsc. 211 (2003) 121-129.

[13] D.W. Hutmacher, Biomaterials 21 (2000) 2529-2543.

[14] J.C. Knowles, G.W. Hastings, J. Mater. Sci. Mater. Med. 3 (1992) 352-358.

[15] H. Li, J. Chang, Biomaterials 25 (2004) 5473-5480.

[16] T. Kokubo, H. Kushitani, S. Sakka, T. Kitsugi, T. Yamamuro, J. Biomed. Mater. Res. 24 (1990) 721-734.

[17] K. James, H. Levene, J.R. Parsons, J. Kohn, Biomaterials 20 (1999) 2203-2212.

[18] B.M.P. Ferreira, C.A.C. Zavaglia, E.A.R. Duek, Mater. Res. 4 (2001) 34-42.

[19] X.H. Zhu, C.H. Wang, Y.W. Tong, J. Biomed. Mater. Res. 89 (2009) 411-423.

[20] L. Ye, J. Chang, C. Ning, K. Lin, J. Biomater. Appl. 22 (2008) 465-480.

[21] H. Li, R. Du, J. Chang, J. Biomater. Appl. 20 (2005) 137-155.

[22] L.J. Chen, M. Wang, Biomaterials 23 (2002) 2631-2639.

[23] H. Li, J. Chang, Polym. Degrad. Stab. 87 (2005) 301-307.

[24] H. Li, W. Zhai, J. Chang, J. Biomater. Appl. 24 (2009) 231-246.

[25] M. Zinn, H.U. Weilenmann, R. Hany, M. Schmid, T. Egli, Acta Biotechnol. 23 (2003) 309-316.

[26] J. Wei, S.J. Heo, D.H. Kim, S.E. Kim, Y.T. Hyun, J.W. Shin, J. R. Soc. Interface 5 (2008) 617-630.

[27] C. Ohtsuki, H. Kushitani, T. Kokubo, S. Kotani, T. Yamamuro, J. Biomed. Mater. Res. 25 (1991) 1363-1370.

[28] A. Bruinink, Serum-free monolayer cultures of embryonic chick brain and retina: immunoassays of developmental markers, mathematical data analysis and establishment of optimal culture conditions, in: G. Zbinden (Ed.), The Brain in Bits and Pieces, MTC Verlag, Zollikon, 1992, pp. 23-30.

[29] A. Bruinink, U. Tobler, M. Hälg, J. Grünert, J. Mater. Sci. Mater. Med. 15 (2004) 497-501.

[30] A. Bruinink, E. Wintermantel, Biomaterials 22 (2001) 2465-2473.

[31] J.P. Kaiser, A. Bruinink, J. Mater. Sci. Mater. Med. 15 (2004) 429-435.

[32] v.P.B. Wachem, C.M. Vreriks, T. Beugeling, J. Feijen, A. Bantjes, J.P. Detmers, W.G.v. Aken, J. Biomed. Mater. Res. 21 (1987) 701-718.

[33] L.L. Hench, J. Am. Ceram. Soc. 81 (1998) 1705-1728.

[34] J.R. Jones, L.L. Hench, Mater. Sci. Technol. 17 (2001) 891-900.

[35] S.K. Misra, S.N. Nazhat, S.P. Valappil, M. Moshrefi-Torbati, R.J. Wood, I. Roy, A.R Boccaccini, Biomacromolecules 8 (2007) 2112-2119.

[36] S.K. Misra, D. Mohn, T.J. Brunner, W.J. Stark, S.E. Philip, I. Roy, V. Salih, J.C. Knowles, A.R. Boccaccini, Biomaterials 29 (2008) 1750-1761.

[37] L.E. Nielsen, R.F. Landel, Mechanical Properties of Polymers and Composites, 2nd ed. CRC Press, 1994.

[38] K. Van de Velde, P. Kiekens, Polym. Test. 21 (2002) 433-442.

[39] F. Gassner, A.J. Owen, Polymer 35 (1994) 2233-2236.

[40] S. Iannace, L. Ambrosio, S.J. Huang, L. Nicolais, J. Appl. Polym. Sci. 54 (1994) 1525-1535.

[41] M. Yasin, B.J. Tighe, Biomaterials 13 (1992) 9-16.

[42] H. Li, W. Zhai, J. Chang, J. Mater. Sci. Mater. Med. 19 (2008) 67-73.

[43] J. Wei, F. Chen, J.W. Shin, H. Hong, C. Dai, J. Su, C. Liu, Biomaterials 30 (2009) 1080-1088.

[44] Y. Arima, H. Iwata, Biomaterials 28 (2007) 3074-3082.

[45] R.Z. Lin, H.Y. Chang, Biotechnol. J. 3 (2008) 1172-1184.

[46] M. Moczulska, M. Bitar, W. Święszkowski, A. Bruinink, J. Biomed, Mater. Res. A 100 (2012) 882-893. 